\title{
UCANS - 8, Paris, July 8-11 th 2019 Eighth International Meeting of the Union of Compact Accelerator-based Neutron Sources
}

\author{
Frédéric Ott ${ }^{1, *}$, Alain Menelle ${ }^{1}$, Christiane Alba-Simionesco ${ }^{1}$ \\ ${ }^{1}$ Laboratoire Léon Brillouin CEA/CNRS, Université Paris Saclay, 91191 Gif sur Yvette Cedex, France
}

\begin{abstract}
Neutron are part of the analytical toolbox for the scientists in a very broad range of fields. Historically, neutrons have been provided by dedicated research reactors. However, the dozens of small and medium size reactors built at laboratories and universities in the previous century are aging. In Europe alone, in 2019, three research reactors have been shut down and it is most unlikely that new research reactors will ever be built in Europe. In the past decades, a number a spallation sources have been built. However, spallation sources are expensive research infrastructures with limited capacity and access.
\end{abstract}

Progress in accelerator technologies as well as neutron moderator technology has opened possibility that more affordable neutron sources might be built using these technologies. In 2010, it was proposed that regular meetings should be organized to share information on Compact Accelerator-based Neutron sources (CANS) using low energy particle accelerators. Since then a number of new projects have emerged across the world and several CANS have been built. The Union of Compact Accelerator-based Neutron Sources was then created.

The $8^{\text {th }}$ international meeting of the Union for Compact Accelerator-Driven Neutron Sources was held in Paris from July 08-11, 2019. More than 140 participants from more than a dozen countries from various continents attended the event. The conference was opened by John Carpenter who retraced the motivations for the creation of the UCANS.

It was shown during the conference that CANS are a viable approach which can potentially offer performances on par with medium power research reactors and spallation sources in the field of neutron scattering. It was shown that simulations suggest high end-CANS can be competitive for SANS, Diffraction or TOF Spectroscopy. This was particularly well illustrated by texture measurements performed at various facilities (RANS@Riken, J-PARC and a medium size reactor); similar quality data could be obtained on the RANS facility operated at a power lower than $1 \mathrm{~kW}$. While the collection time was of course much longer, CANS offer a much easier and fast access for industrialists, students and scientists at a fraction of the cost of large scale facilities.

Hence it is foreseen than CANS could play a significant role in the future neutron landscape. Japan and China are leading the way by building several CANS facilities which will complement their large scale facilities (J-PARC, CSNS, CARR). In the case of Europe, with the foreseen closure of a number of research reactors, CANS projects in France (SONATE) Germany (HBS), Spain, Italy, Hungary.... should also become a key part of the future European Neutron Scattering landscape by providing easy access beam time to users to maintain their current high level of expertise so as to maximize the efficient use of the Large Scale facilities such as ESS and ILL.

The conference covered a very wide range of topics around all aspects of compact sources, accelerators, targets, moderators, detectors, neutron scattering, radiography, isotope and nuclear data, medical applications. The conference Website (ucans8.sciencesconf.org) gathers presentations of the 60 speakers at the conference.

These proceedings show an overview of the current state of development in CANS technologies, prospects and projects.

* Corresponding author: frederic.ott@,cea.fr 



More than 150 participants attended the UCANS-8 conference

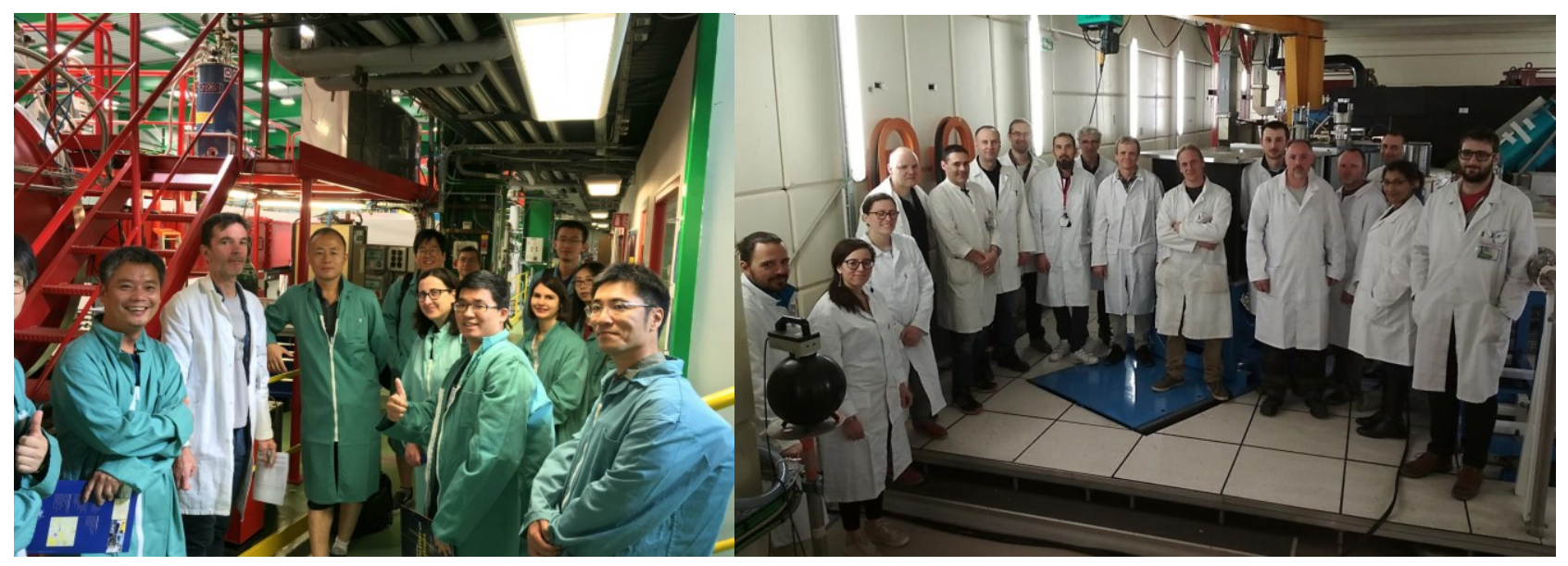

The participants had the opportunity to visit the neutron scattering instruments of the Laboratoire Léon Brillouin at the Orphée reactor (left) and the IPHI - Neutrons accelerator setup (right) in Saclay. 